\section{Switched-Capacitor Algorithmic Digital-to-Analog Converters}

\section{HIROKI MATSUMOTO AND KENZO WATANABE}

Abstract - Novel switched-capacitor circuits for algorithmic digital-toanalog conversion are described. The conversion process is insensitive both

Manuscript received June 28, 1985; revised October 10, 1985.

The authors are with the Research Institute of Electronics, Shizuoka University, 3-5-1 Jyohoku, Hamamatsu 432, Japan.

IEEE Log Number 8608537. to the offset voltages of op-amps and to parasitic capacitances. The capacitance mismatch errors are also minimized because only a small number of unit capacitors are used. An error andysis is presented that shows an accuracy greater than 10-bits can be obtained using present MOS technologies. Besides being very sccurate, the new converters possess the important feature of being integrable using only a minimal amount of chip area.

\section{INTRODUCTION}

Switched-capacitor techniques are very practical for implementing algorithmic digital-to-analog (D/A) converters in a monolithic IC form. Two such circuits have been proposed so far [1]-[3], but they are unfortunately sensitive to parasitic capacitances and/or to the offset voltages of op-amps. This paper describes two switched-capacitor circuits which eliminate these difficulties and thereby allow for high precision $D / A$ conversion.

\section{Conversion Algorithms}

The D/A conversion is a process of transforming a binary number $b$ into an analog voltage $V_{a}$ given by

$$
V_{a}=(-1)^{b_{0}} \sum_{i=1}^{n} 2^{-i} b_{i} V_{r}
$$

where $b_{0}$ is the sign bit which assumes 0 if $b$ is positive and 1 if it is negative, $b_{1}$ and $b_{n}$ are the most significant bit (MSB) and least significant bit (LSB) of $b$, respectively, and $V_{r}$ is the reference voltage. Depending on the bit with which the conversion starts (i.e., $b_{1}$ or $b_{n}$ ) two algorithms are available for the serial D/A conversion. If the conversion starts with $b_{n}$, then $V_{a}$ 


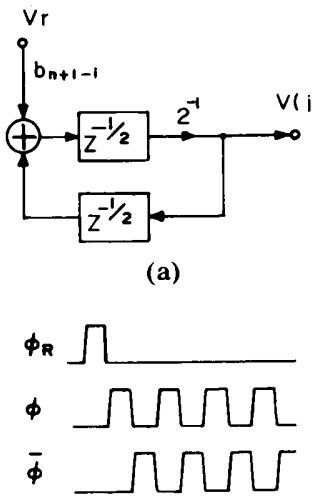

(c)

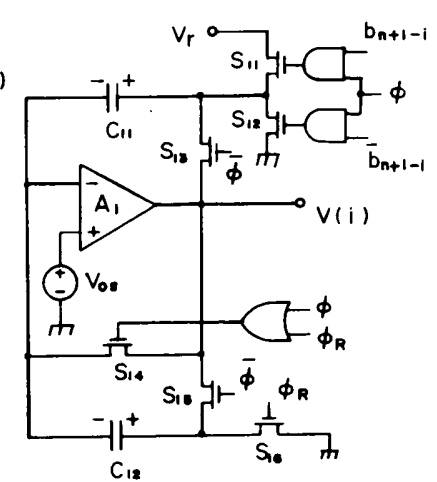

(b)
Fig. 1. The signal flowgraph (a) and the circuit diagram (b) of the DAC-1, and timing diagram of clock signals $(c)$.

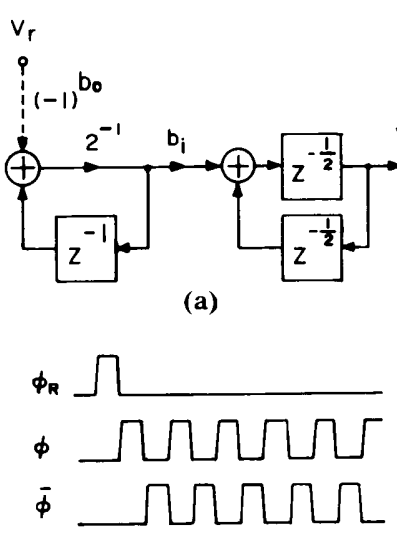

(c)

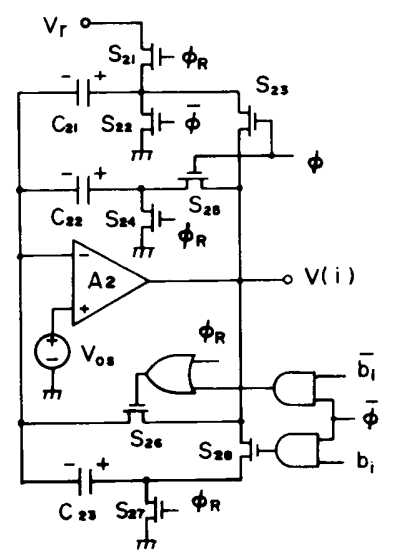

(b)
Fig. 2. The signal flowgraph (a) and the circuit diagram (b) of the DAC-2, and timing diagram of clock signals (c).

can be obtained by iterating the following sequence

$$
V(i)=\left\{V(i-1)+b_{n+1-i} V_{r}\right\} / 2, \quad i=1,2, \ldots, n
$$

where $V(0)=0$ and $V(n)=V_{a}$. The algorithm given by (2), and hence the circuit capable of realizing it, is referred to here as DAC-1. The signal flow graph of DAC-1 is shown in Fig. 1(a), indicating that it can be realized by an integrator followed by a voltage divider which divides the integrator output by 2 .

If the conversion starts with $b_{1}$, on the other hand, $V_{a}$ can be obtained by iterating the following sequence $n$ times:

$$
V(i)=V(i-1)+b_{i} 2^{-i} V_{r}, \quad i=1,2, \ldots, n
$$

where, as before, $V(0)=0$ and $V(n)=V_{a}$. This algorithm for the serial D/A conversion is referred to as DAC-2. The signal flow graph of DAC-2 is shown in Fig. 2(a), which indicates that the DAC- 2 can be realized by a voltage divider which produces the scaled reference voltage followed by an integrator.

\section{Circuit Description}

Fig. 1(b) shows the circuit diagram of DAC-1. The op amp $A_{1}$ and two unit capacitors $C_{11}$ and $C_{12}$ of equal value are used to form the parasitic-insensitive integrator and voltage divider [4], [5]. Prior to the conversion, the reset pulse $\phi_{R}$ is applied to the switches $S_{14}$ and $S_{16}$. This stores the offset voltage $V_{\text {os }}$ of op-amp $A_{1}$ across $C_{12}$. The offset voltage $V_{\text {os }}$ is also stored into $C_{11}$ in the $\phi=$ ' 1 ' phase immediately after $\phi_{R}$. These capacitor voltages practically cancel the offset voltage of op-amp $A_{1}$ in the subsequent conversion period if the op-amp has a reasonably large gain [6], thereby assuring offset-free operation. Therefore, $V_{o s}$ is assumed, without loss of generality, to be negligible henceforth.
TABLE I

SWitching Sequence of DAC-1 for Bipolar CoNversion

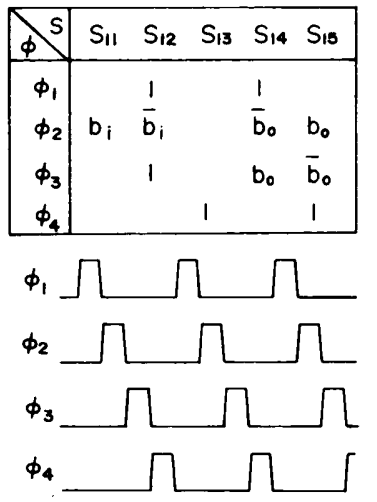

Assume now that the $(i-1)$ th voltage $V(i-1)$ is stored in the capacitor $C_{12}$. Driven by the non-overlapping two phase clock $\phi$ whose timing diagram is shown in Fig. 1(c), the circuit executes the algorithm DAC- 1 as follows: In the $\phi=$ ' 1 ' phase of $i$ th cycle, the capacitor $C_{11}$ is charged to $V_{r}$ by closing $S_{11}$ and $S_{14}$ if $b_{n+1-i}$ is 1 . Otherwise, $C_{11}$ is discharged to 0 by closing $S_{12}$ and $S_{14}$. The voltage across $C_{11}$ is then $b_{n+1-i} V_{r}$. In the next $\phi=$ ' 1 ' phase, $C_{11}$ and $C_{12}$ are connected in parallel. This performs the division by 2 and produces $V(i)$ across $C_{12}$. The circuit repeats this cycle $n$ times, until it converts an $n$-bit word into the desired analog voltage.

Fig. 2(b) shows the circuit diagram of DAC-2. This circuit is also constructed such that the offset voltage of op-amp $A_{2}$ and parasitic capacitances have no effect upon the conversion process. Two unit capacitors $C_{21}$ and $C_{22}$ and op-amp $A_{2}$ form the voltage divider in the $\phi=$ ' 1 ' phase to generate the scaled reference voltage $V_{r} / 2^{i}$, while $C_{21}, C_{23}$, and $A_{2}$ form the integrator in the $\bar{\phi}=$ ' 1 ' phase. Let the voltage across $C_{21}, C_{22}$, and $C_{23}$ be $0, V_{r} / 2^{i-1}$, and $V(i-1)$, respectively, when the $(i-1)$ th cycle of conversion is completed. The algorithm DAC- 2 is executed in one cycle of the non-overlapping two phase clock $\phi$, whose timing diagram is shown in Fig. 2(c), as follows: In the $\phi=$ ' 1 ' phase, the switches $S_{23}$ and $S_{25}$ are "on" to connect $C_{21}$ and $C_{22}$ in parallel. The voltage across $C_{22}$ is then halved because $C_{21}=$ $C_{22}$, to produce $V_{r} / 2^{i}$. In the next $\bar{\phi}={ }^{\prime} 1$ ' phase, the scaled reference voltage $V_{r} / 2^{i}$ thus stored into $C_{21}$ is superposed on $V(i-1)$ stored in $C_{23}$ in the previous cycle if the current bit $b_{i}$ is 1 , or discharged to ground through $S_{26}$ otherwise. The voltages across $C_{21}, C_{22}$, and $C_{23}$ are then $0, V_{r} / 2^{i}$, and $V(i)$, respectively. This cycle is repeated $n$ times, until an $n$-bit word is converted into the analog voltage $V_{a}$.

The above description assumes the unipolar conversion of a positive binary number. A bipolar conversion using only the positive reference voltage is also possible with these circuits. The DAC-1, however, requires a four phase clock. The switching sequence of DAC-1 for the bipolar conversion is listed in Table I. In this table, those switches which are "on" are marked by " 1 " in the corresponding phase. In the $\phi_{2}$ phase, for example, $S_{11}$ is 'on' if $b_{i}$ is 1 and $S_{15}$ is also "on" if the sign bit $b_{0}$ is 1 . Referring to this table, one can identify the circuit operation in each phase; the circuit discharges $C_{11}$ in the $\phi_{1}$ phase, performs the inverting integration if $b$ is negative and the non-inverting integration if $b$ is positive in the $\phi_{2}$ and $\phi_{3}$ phases, and performs the division-by- 2 in the $\phi_{4}$ phase, to resume the above cycle.

The DAC- 2 can convert a negative binary number by the same procedure as that for a positive one, except that, prior to the conversion, the amplifier formed by $C_{21}, C_{22}$, and $A_{2}$ inverts the reference voltage $V_{r}$ and stores it into $C_{22}$. The DAC-2, therefore, accomplishes the bipolar conversion twice as fast as DAC-1. 


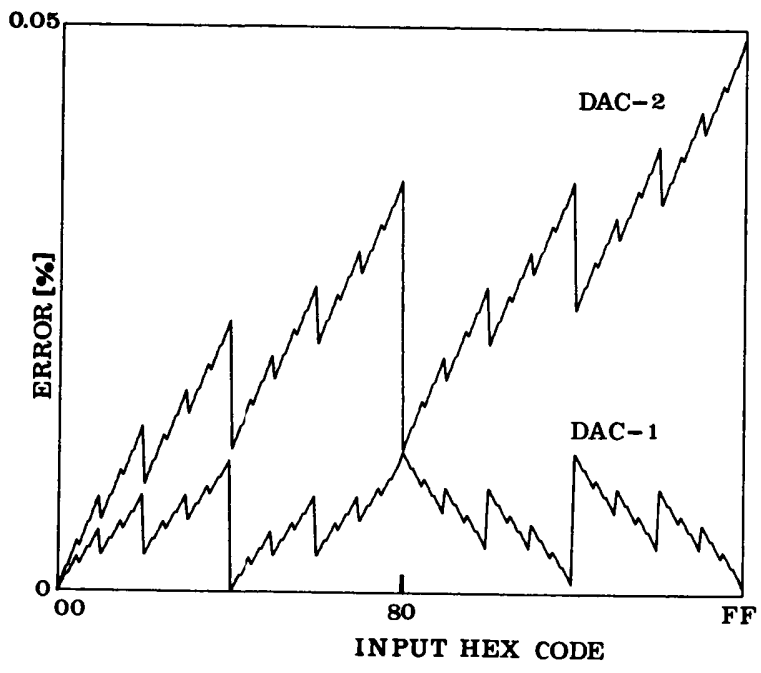

Fig. 3. The errors of the 8-bit DAC-1 and DAC-2 caused by the capacitance mismatch of 0.05 percent.

\section{ACCURACY CONSIDERATIONS}

As described in the previous section, the conversion process of DAC-1 and DAC-2 is insensitive to parasitic capacitances and the offset voltages of op-amps. Therefore, the main error sources are the capacitance mismatch, the clock feedthrough, and the finite open loop gains of op-amps. Their effects upon the conversion accuracy are next estimated separately.

\section{A. Capacitance Mismatch}

Let $\epsilon$ be the capacitance mismatch between $C_{11}$ and $C_{12}$ and between $C_{21}$ and $C_{22}$.

$$
\epsilon=C_{11} / C_{12}-1=C_{22} / C_{21}-1 \text {. }
$$

The conversion algorithm DAC-1 now becomes

$$
V(i)=\frac{V(i-1)+(1+\epsilon) b_{n+1-i} V_{r}}{2+\epsilon} .
$$

Executing (5) $n$ times, the DAC-1 produces the analog voltage

$$
V_{a}^{\prime}=\sum_{i=1}^{n} b_{i}(2+\epsilon)^{-i}(1+\epsilon) V_{r}
$$

The error voltage between $V_{a}$ and $V_{a}^{\prime}$ is then

$$
\Delta V_{\epsilon 1}=\sum_{i=1}^{n} b_{i}\left(2^{-i}-(2+\epsilon)^{-i}(1+\epsilon)\right) V_{r}
$$

Similarly, one can derive the error voltage caused by the capacitance mismatch in the DAC-2;

$$
\Delta V_{\epsilon 2}=\sum_{i=1}^{n} b_{i}\left\{2^{-i}-(2+\epsilon)^{-i}\right\} V_{r}
$$

The errors given by (7) and (8) are plotted in Fig. 3 as a function of the bit pattern of an 8-bit word assuming $\epsilon=0.05$ percent. Inspecting Fig. 3, one notices that the error of DAC-1 becomes maximum when all of the bits except for $b_{1}$ assume 0 and that of DAC-2 becomes maximum when all of the bits assume 1 . For the $n$-bit $\mathrm{D} / \mathrm{A}$ conversion to be accurate down to its LSB, the error should be less than (1/2)LSB. Applying this condition to (7) and (8), we can obtain, to a first order approximation,

$$
\begin{aligned}
2^{n}<1+2 / \epsilon ; & \text { DAC-1 } \\
2^{n+1}-n<2+1 / \epsilon ; & \text { DAC-2 }
\end{aligned}
$$

A capacitance mismatch as small as 0.05 percent is attainable by making unit capacitors moderately large [7]. The accuracy of
DAC-1, limited by the capacitor mismatch, is then estimated to be 12 bits and that of DAC-2 to be 10 bits. These results are consistent with those predicted from Fig. 3. It is noted here that the mismatch between $C_{21}$ and $C_{23}$ of DAC- 2 causes the gain error. This error, however, can be eliminated by transferring the charge accumulated in $C_{23}$ back into $C_{21}$ in the $(n+1)$ th cycle to produce $V_{a}$ [8], [9].

\section{B. Clock Feedthrough}

In the circuit configuration of DAC-1, the switch which causes the most serious clock feedthrough is $S_{14}$, although the other switches are also involved in it. This is also true for $S_{26}$ in the DAC-2. Let $Q_{f}$ be the feedthrough charge which flows through $S_{14}$ towards the inverting input terminal of op-amp $A_{1}$ when $S_{14}$ changes its state from "on" to "off". The conversion algorithm DAC- 1 is then modified to

$$
V(i)=\frac{V(i-1)+b_{n+1-i} V_{r}}{2}+\frac{Q_{f}}{2 C_{12}} .
$$

It is clear from (11) that the error voltage in DAC-1 caused by the feedthrough charge is independent of the bit pattern and takes the value

$$
\Delta V_{f_{1}}=\left(1-2^{-n}\right) Q_{f} / C_{12} .
$$

This error voltage should be again less than (1/2)LSB of an $n$-bit word. Thus one obtains

$$
2^{n+1}<2+C_{12} V_{r} / Q_{f}
$$

The conversion algorithm DAC-2, taking the feedthrough charge from $S_{26}$ into account, is given by

$$
V(i)=V(i-1)+b_{i} 2^{-i} V_{r}+\bar{b}_{i} Q_{f} / 2 C_{23} .
$$

Equation (14) indicates that the error voltage in DAC-2 due to the feedthrough charge becomes maximum when all of the bits assume the value 0 . From the condition that the maximum error should be less than $(1 / 2)$ LSB, one obtains

$$
n \times 2^{n}<C_{23} V_{r} / Q_{f} ; \quad \text { DAC-2. }
$$

A charge ratio $C_{12} V_{r} / Q_{f}\left(C_{23} V_{r} / Q_{f}\right)$ as high as $5 \times 10^{4}$ is possible with presently available MOS technology, if the clock feedthrough cancellation technique is provided [10], [11]. The accuracy limited by the feedthrough effect is then estimated to be 14 bits in DAC-1 and 12 bits in DAC-2.

\section{Finite Op-Amp Gain}

The conversion algorithms when the open-loop gain $A$ of op-amp is finite are given by

$$
\begin{aligned}
& V(i)=\frac{V(i-1)+b_{n+1-i} V_{r} A /(1+A)}{2} ; \text { DAC-1 } \\
& V(i)=V(i-1)+b_{i} 2^{-i} V_{r} A /(1+A) ; \text { DAC-2. }
\end{aligned}
$$

Equations (16) and (17) indicate that the finite gain of op-amp results in the gain or full scale error. For the $n$-bit D/A conversion to be accurate down to its LSB, the gain $A$ should meet the requirement

$$
1+A>2^{n+1} \text {. }
$$

If $A=80 \mathrm{~dB}$, then the accuracy is estimated to be 12 bits.

Summarizing the error analysis, it can be concluded that the most serious error source is the capacitance mismatch and that the accuracy higher than 10 bits can be obtained by the integrated versions of DAC-1 and DAC- 2 . 


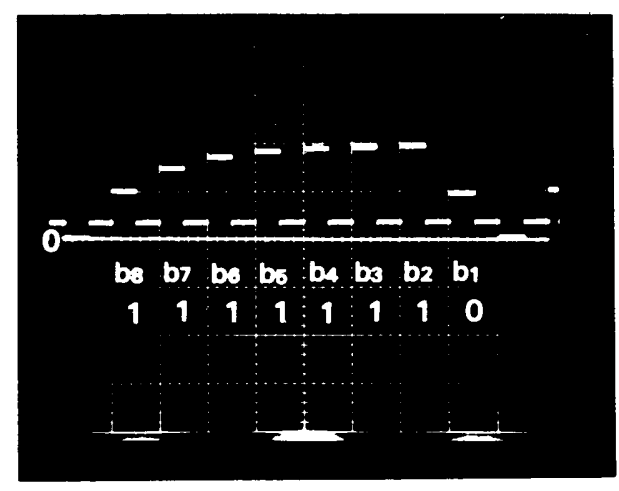

(a)

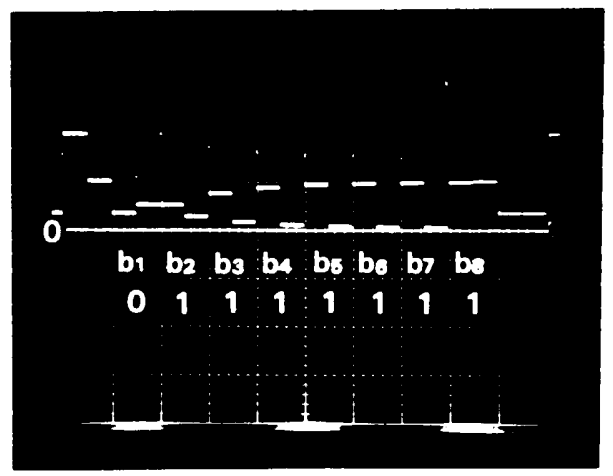

(b)

Fig. 4. Output waveforms of (a) DAC-1 and of (b) DAC-2 observed when the 8-bit word 01111111 was converted into the analog voltage. The reference voltage was $2 \mathrm{~V}$. Horizontal scale: $250 \mu \mathrm{s} /$ div. Vertical scale: $1 \mathrm{~V} /$ div.

\section{EXPERIMENTAL RESULTS}

To confirm the principles of operation, both of the D/A converters were built using discrete components. The op-amps used were LF356 and all of the capacitors used were $1 \mathrm{nF}$. The capacitance mismatch $\epsilon$ was less than 0.5 percent. The reference voltage and the clock frequency were set to $2 \mathrm{~V}$ and $4 \mathrm{kHz}$, respectively. The MOS switches used were $\mathrm{MC14016}$. No clock feedthrough cancellation technique was applied because large capacitors were used for unit capacitors. The signal-to-noise charge ratio $C_{12} V_{r} / Q_{f}\left(C_{23} V_{r} / Q_{f}\right)$ was estimated to be $10^{3}$ by measuring the offset voltage caused by the clock feedthrough. An offset voltage of $0.4 \mathrm{~V}$ was introduced deliberately into the non-inverting input terminal of each op amp.

Figs. 4(a) and (b) show experimentally observed op-amp output waveforms when the 8-bit word 01111111 was converted by DAC-1 and DAC-2, respectively. Each horizontal division, which corresponds to one cycle of the non-overlapping two phase clock, begins with $\bar{\phi}=$ ' 1 ' phase. To identify the operation in each cycle, the bit value being involved in the conversion is also indicated in both figures. Both traces begin with the reset phase and at their leftmost parts the offset voltage applied to the non-inverting input terminals can be seen outputted by the voltage followers $A_{1}$ and $A_{2}$ in the reset phase. Following the offset level, the analog voltage $V(i)$ produced by the serial $\mathrm{D} / \mathrm{A}$ conversion and the offset voltage $0.4 \mathrm{~V}$ can be seen in Fig. 4(a) appearing alternately on the output terminal of op amp $A_{1}$, while the analog voltage $V(i)$ and the scaled reference voltage $V_{r} / 2^{i}$ produced in the $\bar{\phi}=$ ' 1 ' and $\phi=$ ' 1 ' phases, respectively, can be seen in Fig. 4(b) appearing alternately on the output terminal of op-amp $A_{2}$. Despite the very large offset voltage, both DAC-1 and DAC-2 operated satisfactorily and produced the analog voltages of 990 $\mathrm{mV}$ and of $995 \mathrm{mV}$, respectively, in the final cycle. These analog voltages are correct down to 8 bits, which confirms the validity of the error analysis in the previous section.

The clock frequency could be increased up to $100 \mathrm{kHz}$ without causing any degradation in the conversion accuracy. Therefore, the conversion rate up to $10 \mathrm{kHz}$ is possible with these prototype converters.

\section{CONCLUSIONS}

Two algorithms for the serial D/A conversion and their switched-capacitor realizations were presented. Comparing the two circuit designs, the DAC- 1 can be implemented with smaller number of components and is more accurate, while the DAC-2 allows the bipolar conversion at a higher rate. Either design can be easily implemented in IC form using only a very small chip area. Therefore, as low cost and high precision D/A converters, they should find wide applicability for moderate speed data conversion.

\section{ACKNOWLEDGMENT}

Thanks are due to Prof. Ken Martin of the University of California at Los Angeles for critical review and useful comments.

\section{REFERENCES}

[1] R. E. Suárez, P. R. Gray, and D. A. Hodges, "All-MOS charge redistri bution analog-to-digital conversion technique-Part II," IEEE J. SolidState Circuits, vol. SC-10, pp. 379-385, Dec. 1975.

[2] R. H. McCharles, V. A. Saletore, W. C. Black Jr., and D. A. Hodges, "An algorithmic analog-to-digital converter," in IEEE ISSCC Dig. Tech. Paper, pp. 96-97, Feb. 1977.

[3] P. E. Allen and E. Sánchez-Sinencio, Switched-Capacitor Circuits. New York: Van Nostrand, 1984, ch. 7.

[4] K. Martin and A. S. Sedra, "Stray-insensitive switched-capacitor filters based on bilinear z-transform," Electron. Lett., vol. 15, pp. 365-366. June 1979.

[5] F. Maloberti, "Switched-capacitor building blocks for analog signal processing," Electron. Lett., vol. 19, pp. 263-265, Mar. 1983.

[6] R. Gregorian and S. Fan, "Offset free high-resolution D/A converter," in 14th Asilomar Conf. on Circuits, Systems, and Components, pp 316-319, Nov. 1980.

[7] J-B. Shyu, G. C. Temes, and F. Krumenacher, "Random error effects in matched MOS capacitors and current sources," IEEE J. Solid-State Circuits, vol. SC-19, pp. 948-955, Dec. 1984

[8] C-C. Lee, "A new switched-capacitor realization for cyclic analog-todigital converter," in IEEE ISCAS Proc., pp. 1261-1265, Newport Beach, CA, May 1983.

[9] C.-C. Shih, P.-W. Li, and P. Gray, "Ratio independent cyclic A/D and $\mathrm{D} / \mathrm{A}$ conversion using a recirculating reference approach," IEEE Trans. Circuits Syst., vol. CAS-30, pp. 772-774, Oct. 1983.

[10] K. Martin, "New clock feedthrough cancellation technique for analog MOS switched-capacitor circuits," Electron. Lett., vol. 18, pp. 39-40, Jan. 1982.

[11] P. W. Li, M. J. Chin, P. R. Gray, and R. Castello, "A ratio-independent algorithmic analog-to-digital conversion technique," IEEE J. Solid-State Circuits, vol. SC-19, pp. 828-836, Dec. 1984

\section{A Unified Study on the Roundoff Noise in 2-D State Space Digital Filters}

\author{
TAO LIN, MASAYUKI KAWAMATA, \\ AND TATSUO HIGUCHI
}

Abstract - This paper studies the roundoff noise and the scaling in 2-D state space digital filters. The noise matrix and the covariance matrix in the 2-D case are derived. They are expressed with double infinite sums or

Manuscript received August 13, 1985; revised December 9, 1985

The authors are with the Department of Electronic Engineering, Faculty of Engineering, Tohoku University, Aoba, Aramaki, Sendai 980, Japan. IEEE Log Number 8608539. 